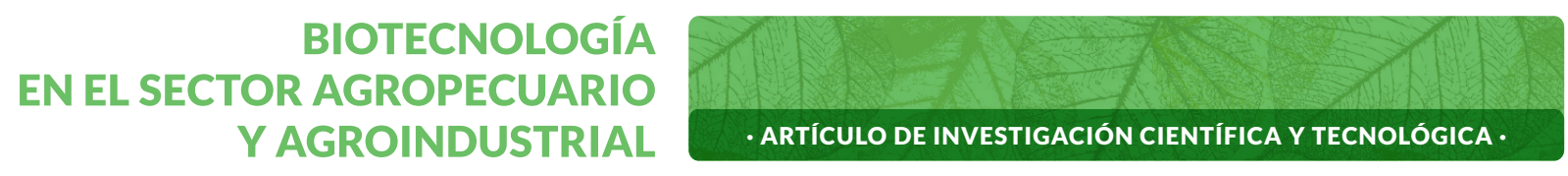

\title{
Efecto crioprotector de la yema de huevo en semen de Prochilodus mariae (Characiformes: Prochilodontidae)*
}

\section{Cryoprotective effect of egg yolk in semen of Prochilodus mariae (Characiformes: Prochilodontidae)}

\section{Efeito cioprotetor do iêm de ovo no sêmen de Prochilodus mariae (Characiformes: Prochilodontidae)}

DUARTE-TRUJILLO, ASTRID-STEFANÍA'; ARDILA-ARTUNDUAGA, MICHELLE-BRIGITTE²; GUAJE-RAMÍREZ, DIANA NATHALIE'; MEDINA-ROBLES, VÍCTOR-MAURICIO ${ }^{4}$

Historial del Artículo

Recibido para evaluación: 14 de Julio 2020.

Aprobado para publicación: 20 de Octubre 2020

* Título del proyecto de origen: "Evaluación de protocolos de crioconservación seminal en especies ícticas nativas como línea base para la conformación de un banco de semen de peces nativos con fines comerciales y de conservación". Financiación: Gobernación del Meta; Ministerio de Ciencia, Tecnología e Innovación; Universidad de los Llanos. Culminación: agosto de 2020.

1 Universidad de los Llanos, Facultad de Ciencias Agropecuarias y Recursos Naturales (FCARN), Instituto de Acuicultura de los Llanos, Grupo de Investigación Sobre Reproducción y Toxicología de Organismos Acuáticos (GRITOX). Ingeniero Agroindustrial. Villavicencio, Colombia. https://orcid.org/0000-0003-1777-374X

2 Universidad de los Llanos, Facultad de Ciencias Agropecuarias y Recursos Naturales (FCARN), Programa de Medicina Veterinaria y Zootecnia. Estudiante. Villavicencio, Colombia. https://orcid.org/0000-0002-0013-0003

3 Universidad de los Llanos, Facultad de Ciencias Agropecuarias y Recursos Naturales (FCARN), Instituto de Acuicultura de los Llanos, Grupo de Investigación Sobre Reproducción y Toxicología de Organismos Acuáticos (GRITOX). Médico Veterinario y Zootecnista. Villavicencio, Colombia. https://orcid.org/0000-0001-6519-6372

4 Universidad de los Llanos, Facultad de Ciencias Agropecuarias y Recursos Naturales (FCARN), Instituto de Acuicultura de los Llanos, Grupo de Investigación Sobre Reproducción y Toxicología de Organismos Acuáticos (GRITOX). Dr. (c) en Ciencias Agrarias. Villavicencio, Colombia. https://orcid.org/0000-0002-4871-2715 


\section{RESUMEN}

Prochilodus mariae es una especie endémica del río Orinoco, de gran importancia ecológica, cultural y económica, cuyo protocolo de crioconservación seminal aún no ha sido establecido. El objetivo de este trabajo fue evaluar el efecto del uso y la exclusión de la yema de huevo en el diluyente de crioconservación, sobre la calidad seminal de P. mariae. Para ello, el semen fresco de cinco machos sanos y sexualmente maduros fue diluido (1:6), empacado en pajillas (0,5 mL), congelado y almacenado en nitrógeno líquido. Se emplearon dos tratamientos: diluyente con yema de huevo (YH) y sin yema de huevo (SYH). Se evaluó motilidad, duración de la motilidad, morfología, integridad de membrana plasmática y fertilidad del semen descongelado, usando como control semen fresco. Los datos fueron tratados con estadística descriptiva y paramétrica, con un nivel de confianza del 95\%. Los resultados demostraron que la exclusión de la yema de huevo mejoró significativamente la motilidad y la duración de la motilidad, aunque no presentó una influencia estadísticamente significativa sobre la integridad de membrana plasmática, la anormalidad morfológica y la fertilidad. La adición de la yema de huevo al diluyente de crioconservación no presentó el efecto crioprotector esperado, siendo necesario evaluar diferentes concentraciones e interacciones con otros componentes del diluyente.

\section{ABSTRACT}

Prochilodus mariae is an endemic species from Orinoco River, of great ecological, cultural and economic importance, whose seminal cryopreservation protocol has not yet been established. The aim of this work was to evaluate the effect of use and exclusion of egg yolk in the cryopreservation diluent, on the seminal quality of $P$. mariae. Thus, fresh semen of five healthy and sexually mature males was diluted (1:6), packed in 0,5 $\mathrm{mL}$ straws, frozen and stored in liquid nitrogen. Two treatments were used: diluent with egg yolk (YH) and diluent without egg yolk (SYH). Motility, motility duration, morphology, plasma membrane integrity and fertility of thawed semen were evaluated, using fresh semen as control. The data were treated with descriptive and parametric statistics, with a confidence level of $95 \%$. The results showed that exclusion of egg yolk significantly improved motility and motility duration, although it did not have a statistically significant influence on plasma membrane integrity, morphological abnormality and fertility. The addition of the egg yolk to the cryopreservation diluent did not present the expected cryoprotective effect. It's necessary to evaluate different concentrations of egg yolk and its interactions with other components of the diluent.

Correspondencia:vmmedinarobles@unillanos.edu.co

Cómo citar este artículo: DUARTE-TRUJILLO, ASTRID-STEFANÍA; ARDILAARTUNDUAGA, MICHELLE-BRIGITTE; GUAJE-RAMÍREZ, DIANA-NATHALIE; MEDINA-ROBLES, VÍCTOR-MAURICIO. Efecto crioprotector de la yema de huevo en semen de Prochilodus mariae (Characiformes: Prochilodontidae). Biotecnología en el sector agropecuario y agroindustrial, v. 19, n. 1, 2021, p. 191-205. Doi: https://doi. org/10.18684/BSAA(19)191-205

\section{PALABRAS CLAVE:}

Bocachico; Calidad seminal; Crioconservación; Espermatozoide; Characiformes: Prochilodontidae.

\section{KEYWORDS:}

Bocachico fish; Seminal quality; Cryopreservation; Spermatozoa; Characiformes: Prochilodontidae.

\section{PALAVRAS-CHAVE:}

Coporo; Qualidade seminal; Criopreservação; Espermatozóide; Characiformes: Prochilodontidae. 


\section{RESUMO}

Prochilodus mariae é uma espécie endêmica do rio Orinoco, de grande importância ecológica, cultural e econômica, cujo protocolo de criopreservação seminal ainda não foi estabelecido. O objetivo deste trabalho foi avaliar o efeito do uso $e$ exclusão da gema de ovo no diluente de criopreservação, na qualidade seminal de P. mariae. Para isso, o sêmen fresco de cinco machos saudáveis e sexualmente maduros foi diluído (1:6), acondicionado em palhinhas (0,5 mL), congelado e armazenado em nitrogênio líquido. Foram utilizados dois tratamentos: diluente com gema de ovo (YH) e diluente sem gema de ovo (SYH). Avaliou-se motilidade, duração da motilidade, morfologia, integridade da membrana plasmática e fertilidade do sêmen descongelado, utilizando sêmen fresco como controle. Os dados foram tratados com estatística descritiva e paramétrica, com nível de confiança de $95 \%$. Os resultados mostraram que a exclusão da gema de ovo meIhorou significativamente a motilidade e a duração da motilidade, embora não tenha tido influência estatisticamente significativa na integridade da membrana plasmática, anormalidade morfológica e fertilidade. A adição da gema de ovo ao diluente de criopreservação não apresentou o efeito crioprotetor esperado. E necessário avaliar diferentes concentrações gema de ovo e suas interações com outros componentes do diluente.

\section{INTRODUCCIÓN}

Pese a ocupar menos del $1 \%$ de la superficie terrestre, los peces de agua dulce proveen cerca del $10 \%$ de la biodiversidad de especies descritas y representan alrededor de una cuarta parte de los vertebrados del mundo [1, 2]. Sin embargo, los peces son más sensibles que los organismos terrestres a los cambios en el hábitat [3]. Se estima que las tasas de extinción de los peces dulceacuícolas son entre 112 a 855 veces más altas que las tasas de extinción natural [4]. Tan solo en Colombia, la segunda nación sudamericana más biodiversa en peces de agua dulce, con 1500 especies registradas, se reportan 81 especies con algún grado de riesgo de extinción [5, 6].

La acuicultura en Colombia, es una actividad económica que ha ido en ascenso en los últimos años, dada su mayor rentabilidad en contraste con las actividades agropecuarias tradicionales, y la disminución significativa de la pesca de captura a causa de la sobreexplotación. El sector acuicultura está dominado en gran medida por la piscicultura de agua dulce interior; para el año 2017 se reportaron cerca de 120.000 toneladas de productos pesqueros, siendo el departamento del Huila el principal productor, con un aporte de $46 \%$ de la producción, seguido del departamento del Meta, con un aporte del 13\% [7-9].

Prochilodus mariae Eigenmann 1922 (Characiformes: Prochilodontidae), también conocido como bocachico llanero o coporo, es una de las 13 especies del género Prochilodus. Es un pez migratorio y endémico de la cuenca del río Orinoco, de gran importancia ecológica, cultural y económica. Es una de las especies de la cuenca del Orinoco más explotada por las pesquerías de Colombia y Venezuela [10,11]. Su explotación se limita a la pesca de captura, debido a que su cultivo comercial presenta bajo desempeño productivo en cuanto a la relación de la contribución de la dieta y la ganancia de peso [12]. Aunque el bocachico llanero ha sido explotado por más de 50 años, aún no se encuentra categorizado dentro de las especies en riesgo de extinción [6,13].

Para preservar la diversidad e integridad genética del bocachico llanero, existen herramientas biotecnológicas como la crioconservación, que consiste en el almacenamiento de células viables a bajas temperaturas y largo término. El tipo de célula más crioconservada son los espermatozoides, ya que al ser relativamente más resistentes a la congelación y ocupar menos espacio, facilitan tanto su manipulación in vitro como su transporte desde y hacia las fincas, permitiendo el suministro constante de gametos masculinos durante cualquier época del año [14-16] Junto con las tecnologías reproductivas, la crioconservación juega también un papel clave en los programas de mejora genética e investigación científica [17], constituyendo una alternativa viable para afrontar el bajo desempeño productivo del bocachico llanero, que ha limitado su cultivo comercial [12], aunque es sabido que, este tipo de estrategias en peces neotropicales todavía es incipiente [17]. 
Infortunadamente, la crioconservación induce daños celulares mediante diferentes mecanismos, tales como la formación de cristales de hielo, el estrés osmótico, el estrés oxidativo, entre otros, los cuales repercuten en la fertilidad seminal [18]. En consecuencia, se han incorporado crioprotectores tanto internos como externos al diluyente de crioconservación, con el fin de incurrir en el menor daño crioinducido y promover la supervivencia celular [19]. Los crioprotectores internos, es decir, los que penetran a la célula, son los más efectivos, aunque se ha reportado que pueden llegar a ser tóxicos [20]. Por su parte, los crioprotectores externos (crioprotectores no penetrantes) son inocuos y juegan un rol importante como estabilizadores de membrana [21]. La yema de huevo es un crioprotector externo ampliamente empleado, ya que tiene en su composición lipoproteínas de baja densidad (LDL) que se adhieren a la membrana celular y la protegen del daño mecánico a causa de la formación de cristales de hielo en el exterior [22].

Para garantizar la máxima protección celular contra el daño criogénico, debe existir un protocolo de crioconservación seminal para cada especie en particular, ya que las características espermáticas difieren entre especies y, por ende, el efecto de cada uno de los parámetros de operación del protocolo sobre la calidad seminal es variable [15]. Sin embargo, existe limitado conocimiento con respecto a los protocolos de crioconservación en especies del género Prochilodus [19]. En este contexto, el objetivo del trabajo es evaluar el efecto del uso y la exclusión de la yema de huevo del diluyente de crioconservación, sobre la calidad seminal de Prochilodus mariae.

\section{MÉTODO}

Todos los procedimientos fueron realizados de conformidad con las normas y procedimientos para el uso de animales de laboratorio, descritas por el Comité on Care and Use of Laboratory Animal Resources - National Research Counsil, USA (1996).

\section{Área de estudio}

Los experimentos fueron realizados durante el periodo comprendido entre abril y junio de 2019, en el Laboratorio de Reproducción y Crioconservación del Instituto de Acuicultura de la Universidad de los Llanos, localizado en la ciudad de Villavicencio (Meta, Colombia) en las coordenadas geográficas 404'24.5”N 73³4'55.3”W, a una altitud de 418 msnm, temperatura ambiental media de $27^{\circ} \mathrm{C}$, humedad relativa del $75 \%$ y precipitación pluvial de $4050 \mathrm{~mm} /$ año.

\section{Material biológico}

Se utilizaron reproductores adultos (machos y hembras) de Prochilodus mariae con peso corporal de 0,45 $\pm 0,02$ $\mathrm{kg}$ y $0,92 \pm 0,11 \mathrm{~kg}$, y longitud total de $32,5 \pm 0,85 \mathrm{~cm}$ y $38,5 \pm 1,75 \mathrm{~cm}$, respectivamente. Los peces fueron nacidos y criados en cautiverio en la Estación Piscícola de la Universidad de los Llanos, alimentados una vez al día, cinco veces por semana, a razón del 3\% del peso vivo con pellets extruídos (24-45\% de proteína bruta, dependiendo de la etapa de desarrollo).

La selección de los ejemplares se hizo con base en su madurez sexual y desarrollo corporal. La madurez sexual de los machos fue determinada por la presencia de semen en la papila urogenital después de realizar presión sobre el abdomen, mientras que en las hembras se determinó por biopsia ovárica y evaluación del estado de desarrollo de los ovocitos en estereoscopio Nikon SMZ800 (Nikon Instruments Inc., Melville, NY) [23]. Se seleccionaron las hembras con diámetros de ovocitos uniformes, mayor proporción de ovocitos con vesícula germinal migrando y mayor número de ovocitos viables [24, 25]. Los ovocitos viables se definieron como traslúcidos, perfectamente esféricos y sin espacio vitelino [26].

Los animales seleccionados fueron transferidos a una pileta circular de $7000 \mathrm{~L}$, con recambio de agua, aireación permanente y sin alimentación. Inmediatamente, fueron caracterizados (peso, longitud estándar y longitud to- 
tal) e identificados con esferas de colores adheridas a la aleta dorsal. Se mantuvieron allí durante el período de inducción hormonal hasta la espermiación y desove (aproximadamente 32 h).

\section{Inducción hormonal}

La maduración final de las gónadas se provocó mediante administración intramuscular de Extracto de Pituitaria de Carpa (Cyprinus carpio) - EPC (Argent Aquaculture, Redmond, WA, USA) en la base de la aleta dorsal. La ovulación fue inducida con una dosis total de $5,5 \mathrm{mg} / \mathrm{kg}$ de peso corporal, distribuida en dos aplicaciones con intervalo de 12 horas ( $0,5 \mathrm{mg} / \mathrm{kg}$ y $5,0 \mathrm{mg} / \mathrm{kg}$ de peso corporal). La espermiación fue inducida con una única dosis de $4 \mathrm{mg} / \mathrm{kg}$ de peso corporal, aplicada simultáneamente con la primera dosis de inducción de las hembras [27].

\section{Obtención de gametos}

Los ejemplares fueron anestesiados por inmersión en 2-Fenoxi-etanol (366 ppm; FLUKA, Sigma-Aldrich Co., St. Louis, MO, USA) hasta la pérdida del eje de nado. Posteriormente, se removieron de la solución anestésica, se secó cuidadosamente la papila urogenital y se extrajeron los gametos mediante masaje abdominal en sentido cráneo-caudal. Los ovocitos fueron colectados en recipientes de plástico limpios y secos, mientras que el semen fue colectado en tubos de ensayo graduados y estériles. Para determinar la cantidad de material obtenido, el peso $(\mathrm{kg})$ del desove se calculó en balanza digital Traveler TA501 (Ohaus Co., Pine Brook, NJ, USA) y el volumen $(\mathrm{mL})$ de esperma se midió directamente en el tubo de ensayo. Las muestras contaminadas con heces, sangre $\mathrm{u}$ orina fueron descartadas [28,29]. La eyaculación y el desove fueron realizados aproximadamente 7-8 h después de la última dosis hormonal en la hembra.

\section{Crioconservación}

Los diluyentes fueron preparados usando cloruro de sodio ( $\mathrm{NaCl} 0,9 \% \mathrm{p} / \mathrm{v})$ como solución base. El diluyente sin yema de huevo (SYH) se preparó con $6 \%$ p/v de glucosa $\left(\mathrm{C}_{6} \mathrm{H}_{12} \mathrm{O}_{6}\right.$; Sigma-Aldrich, St. Louis, MO, USA) y $10 \%$ v/v de metanol ( $\mathrm{CH}_{3} \mathrm{OH}$; Merck KGaA, Darmstadt, Germany). El diluyente con yema de huevo (YH) tuvo la misma concentración de glucosa y metanol que $\mathrm{SYH}$, pero incluyó adicionalmente $12 \% \mathrm{v} / \mathrm{v}$ de yema de huevo, sin membrana vitelina. Los componentes se adicionaron en el mismo orden de mención [30].

El semen fresco fue sometido a un análisis de calidad seminal y posteriormente, fue diluido en proporción 1:6 con cada diluyente y empacado en pajillas francesas de 0,5 mL (Instrument de Médecine Vétérinaire, Minneapolis, MN, USA). Luego de esto, las pajillas fueron congeladas en vapores de nitrógeno (Dry Shipper CX100; Taylor-Wharton, Theodore, AL, USA) a $-70^{\circ} \mathrm{C}$ durante $30 \mathrm{~min}$ y almacenadas en nitrógeno líquido a $-196^{\circ} \mathrm{C}$ (Deward 35 HC; Taylor-Wharton, Theodore, AL, USA) durante siete días [23, 27, 29].

Para la evaluación de la calidad seminal post-crioconservación, las pajillas fueron descongeladas en baño maría a $37^{\circ} \mathrm{C}$ durante 60 segundos (CITO products Inc., Watertown, WIS, USA). El semen descongelado fue vertido en tubos de ensayo graduados y estériles.

\section{Evaluación de la calidad seminal}

La calidad seminal se evaluó tanto en semen fresco como en semen descongelado.

Análisis cualitativo. Se registró el volumen del semen fresco obtenido y se observó la coloración para descartar una posible contaminación con heces, sangre u orina. El color ideal del semen es blanco puro o marfil [23].

Motilidad espermática. Se estimó subjetivamente mediante observación en microscopio convencional Axiostar (Carl Zeiss LLC, Thornwood, NY, USA) con un aumento de 100X, previa dilución 1:20 en solución activadora, así: 
agua destilada para semen fresco y bicarbonato de sodio $1 \%\left(\mathrm{NaHCO}_{3}\right.$, Merck KGaA, Darmstadt, Germany) para semen descongelado. La motilidad se expresó como porcentaje de espermatozoides móviles en la muestra. Las muestras de semen fresco con movilidades inferiores al $80 \%$ no fueron procesadas [23, 29].

Duración de la motilidad. Se midió con un cronómetro digital, contabilizando el tiempo transcurrido en segundos, desde la activación de los espermatozoides hasta que aproximadamente el $90 \%$ de los espermatozoides dejó de moverse.

Integridad de membrana plasmática. Fue evaluada por el método de tinción con eosina 1,1\% (C.I. 45400; Sigma-Aldrich, St. Louis, MO, USA) - nigrosina 5,3\% (C.I. 50420, Merck KGaA, Darmstadt, Germany) - citrato de sodio 2,9\% (Sigma-Aldrich, St. Louis, MO, USA) [31]. Se realizó un extendido en lámina portaobjeto (Microscope slides, Ground edges) utilizando $5 \mu \mathrm{L}$ de semen y $45 \mu \mathrm{L}$ del colorante. Luego, se analizó por microscopía óptica 100X (Primo Star, Carl Zeiss LLC, Göttingen, Germany). La integridad de membrana plasmática se expresó como porcentaje de células viables (vivas), las cuales se identificaron por permanecer incoloras. Las células teñidas correspondieron a células con integridad de membrana comprometida [32].

Concentración espermática. Fue determinada por microscopía óptica 100X (Primo Star, Carl Zeiss LLC, Göttingen, Germany) mediante recuento en cámara de Neubauer mejorada (0,1 mm de profundidad; Paul Marienfeld GmbH \& Co, Lauda-Königshofen, Germany), previa dilución 1:20 en solución fijadora formol - citrato ( $4 \% \mathrm{v} / \mathrm{v} \mathrm{CH}_{2} \mathrm{O} 37 \%$ y $2,9 \% \mathrm{p} / \mathrm{V} \mathrm{Na}_{3} \mathrm{C}_{6} \mathrm{H}_{5} \mathrm{O}_{7} ;$ Merck KGaA, Darmstadt, Germany). La concentración se expresó como millones de espermatozoides por microlitro (spz/ $\mu \mathrm{L})[23,33]$.

Morfología. Se analizó mediante tinción con rosa de bengala 3\% (Alfa Aesar, Tewksbury, MA, USA). Para ello, el semen fue fijado 1:1000 en solución formol - citrato (4\% v/v CH $\mathrm{O}_{2} \mathrm{O} 3 \%$ y 2,9\% p/ $/ \mathrm{Na}_{3} \mathrm{C}_{6} \mathrm{H}_{5} \mathrm{O}_{7}$; Merck KGaA, Darmstadt, Germany) y almacenado en refrigeración a $4^{\circ} \mathrm{C}$ hasta su uso [31]. Previo al análisis, las muestras de semen fijado fueron homogenizadas mediante Vortex (Fisher Scientific ${ }^{\mathrm{TM}}$ Digital Vortex Mixer, EEUU). Posteriormente, se colocaron $15 \mu \mathrm{L}$ de la mezcla homogenizada sobre una lámina portaobjeto, se agregaron $0,5 \mu \mathrm{L}$ de Rosa de Bengala 3\%, se homogenizó y se realizó un frotis delgado. Se analizó la morfología de los espermatozoides por microscopía óptica 100X (Primo Star, Carl Zeiss LLC, Göttingen, Germany). Se evaluaron las anormalidades secundarias descritas por Miliorini et al. [34]. Los resultados se expresaron como porcentaje de células anormales.

Fertilidad. Se seminaron 2,0 g de desove de una única hembra con $400 \mu \mathrm{L}$ de semen descongelado para cada uno de los tratamientos y $100 \mu \mathrm{L}$ de semen fresco para el control (semen de un único ejemplar sano y sexualmente maduro). Los espermatozoides fueron activados con la adición de $5 \mathrm{~mL}$ de bicarbonato de sodio $1 \%$ y agua, para los tratamientos y el control, respectivamente. Se removió la mezcla durante un minuto. Posteriormente, se lavó con abundante agua y se incubó conforme a lo descrito por Velasco-Santamaría et al. [30]. Durante la incubación, se monitorearon los parámetros físicoquímicos del agua por medio de una sonda multiparamétrica ( $\mathrm{HACH}$ HQ40D, Loveland, CO, USA). Luego de cinco horas de incubación, se tomaron muestras de ovocitos de cada uno de los tratamientos. La fertilidad se estableció como el porcentaje de ovocitos embrionados [29]. Los ovocitos embrionados se identificaron por ser esféricos y traslúcidos, en contraste con los no fertilizados, que presentan coloración blanquecina y opaca [26]. Todos los tratamientos se realizaron por triplicado.

\section{Diseño experimental}

Se aplicó un Diseño Completamente al Azar (DCA). Se evaluaron dos tratamientos experimentales y un control (semen fresco), con cinco réplicas cada uno, donde cada macho fue considerado como una réplica. El factor de estudio fue el diluyente de crioconservación, con dos niveles: yema de huevo (YH), sin yema de huevo (SYH). Las variables de respuesta analizadas fueron los parámetros de calidad post-congelación: motilidad (\%), duración de la motilidad (s), integridad de membrana plasmática (\%), anormalidad (\%) y fertilidad (\%). Los valores obtenidos 
en semen fresco fueron usados como control, a excepción de la prueba de fertilidad (\%F), para la cual se empleó el semen de otro ejemplar sano y sexualmente maduro.

\section{Análisis estadístico}

Los datos obtenidos fueron organizados y procesados mediante estadística descriptiva, y expresados como media \pm error estándar (SEM, por sus siglas en inglés). El efecto de los tratamientos en la calidad seminal se determinó mediante análisis de varianza de una vía (ANOVA), con un nivel de confianza del 95\%, luego de validación de los supuestos de la estadística paramétrica: homocedasticidad (prueba de Bartlett) y normalidad de los datos (prueba de Shapiro-Wilk). Datos que no cumplieron con los supuestos, se analizaron mediante la prueba no paramétrica de Kruskal-Wallis. Se empleó el software estadístico GraphPad Prism 8XML para Windows (https://www.graphpad.com/)

\section{RESULTADOS}

\section{Caracterización de semen fresco}

Se obtuvo, en promedio, 1,1 $\pm 0,24 \mathrm{~mL}$ de semen por macho $(\mathrm{n}=5)$. El semen colectado presentó una coloración blanca y una concentración promedio de $26,179 \pm 2,773 \mathrm{spzx} 10^{6} / \mu \mathrm{L},(\mathrm{spz}=$ espermatozoides).

\section{Caracterización de ovocitos}

De la hembra seleccionada se obtuvieron $130 \mathrm{~g}$ de desove con una concentración de 2177 ovocitos/g y diámetro ovocitario promedio de $944,4 \pm 15,95 \mu \mathrm{m}(n=30)$. La muestra aleatoria $(n=50)$ de ovocitos presentó el $52 \%$ con núcleos migrando (maduros), el $28 \%$ centrales (inmaduros), el $2 \%$ periféricos (sobremaduros) y el $18 \%$ atrésicos (inviables).

\section{Caracterización del agua de incubación}

El agua de incubación presentó las siguientes características fisicoquímicas: $\mathrm{pH}$ de 6,5 \pm 0,007; temperatura de $25,5 \pm 0,124^{\circ} \mathrm{C}$; saturación de oxígeno del $85,8 \pm 0,709 \%$; oxígeno disuelto de $6,7 \pm 0,032 \mathrm{mg} \mathrm{O}_{2} / \mathrm{L}$; y conductividad de $70,1 \pm 9,265 \mu \mathrm{S} / \mathrm{cm}$.

\section{Calidad seminal}

La figura 1 muestra el efecto de los tratamientos YH y SYH sobre los parámetros de calidad seminal post-congelación en P. mariae, usando semen fresco como control.

Se evidencia que la crioconservación per se, influye negativa y significativamente sobre la motilidad, la integridad de membrana plasmática y la normalidad espermática, siendo ésta última la más notoria. Los tratamientos sin yema de huevo $(\mathrm{SYH})$ y yema de huevo $(\mathrm{YH})$ presentaron diferencias estadísticamente significativas entre sí, en cuanto a su efecto sobre la motilidad y la duración de la motilidad (Figuras 1-A y 1-B). Con el tratamiento $\mathrm{SYH}$ se obtuvieron los mayores valores promedio de motilidad $(32 \pm 3,7 \%$ ) y duración de la motilidad ( $56 \pm 2,3$ s), en contraste con el tratamiento $\mathrm{YH}$, que permitió tan sólo el $11 \pm 2,4 \%$ de motilidad con una duración de 43 $\pm 2,5$ segundos. En cuanto a la morfología, pese a no haber diferencias estadísticamente significativas entre tratamientos, el porcentaje medio de anormalidades disminuyó al omitirse la yema de huevo del diluyente de crioconservación, pasando del $80 \pm 2,7 \%$ en YH al $72 \pm 6,0 \%$ en SYH (Figura 1-D). En cuanto a la integridad de membrana plasmática tampoco hubo diferencias estadísticamente significativas entre tratamientos, aunque con la incorporación de la yema de huevo aumentó del $72 \pm 3,4 \%$ en SYH al $80 \pm 2,7 \%$ en YH (Figura 1-C). La integridad de membrana plasmática fue la única variable que mejoró con el tratamiento $\mathrm{YH}$. 
Figura 1. Evaluación de parámetros de calidad seminal post-congelación en Prochilodus mariae, empleando dos diluyentes de crioconservación ( $\mathrm{YH}=$ con yema de huevo; $\mathrm{SYH}=$ sin yema de huevo). Control $=$ semen fresco. Parámetros $=(A)$ Motilidad, $(B)$ Duración de la motilidad, $(C)$ integridad de membrana plasmática, (D) Anormalidad. Valores expresados como media $\pm \mathrm{SEM}(n=5)$. Entre barras, letras distintas indican diferencia estadística $(P<0,05)$.

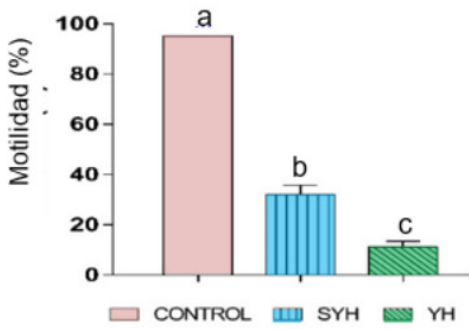

A.

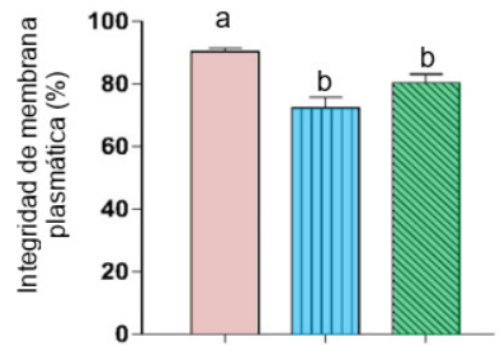

C.

$\square$ CONTROL $\amalg$ SYH

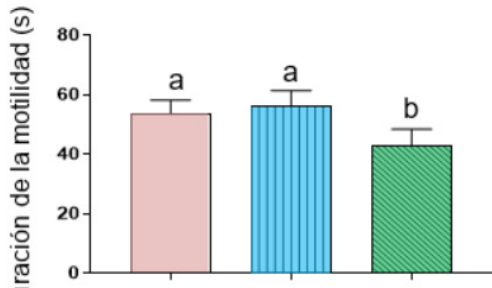

B.

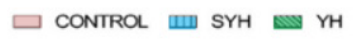

La figura 2 muestra el efecto de los tratamientos YH y SYH sobre la fertilidad seminal post-congelación en $P$. mariae, empleando como control el semen fresco de otro macho sano y sexualmente maduro. Se evidencia que la omisión de la yema de huevo (12\% v/v) en el diluyente de crioconservación, no influyó significativamente sobre la fertilidad post-congelación de P. mariae, ya que no hubo diferencias estadísticamente significativas entre los tratamientos. Sin embargo, los valores de fertilidad aumentaron de $13,6 \pm 5,7 \%$ en el tratamiento $\mathrm{YH}$ al $29,5 \pm$ $4,5 \%$ en el tratamiento $\mathrm{SYH}$ (Figura 2 ).

En este orden de ideas, la adición 12\% de yema de huevo al diluyente de crioconservación no mejoró la calidad seminal de P. marie; por el contrario, limitó la motilidad y la duración de la motilidad. Se esperaba que la incorporación de yema de huevo al diluyente de crioconservación mejorara la calidad seminal post-congelación de $P$. mariae, ya que está reportado que la yema de huevo se compone de sustancias con actividad crioprotectora demostrada, tales como los fosfolípidos y las lipoproteínas de alta densidad (HDL, por sus siglas en inglés) [16, 35]. Según Dong et al. [36], no debería existir diferencias significativas entre el efecto crioprotector de la yema de huevo y el efecto crioprotector de sus componentes aislados (fosfolípidos y HDL). Inclusive, según varios autores, la yema de huevo es por sí sola, un protector y estabilizador de membrana probado en varias especies, incluidos los peces [37, 38].

El efecto de la yema de huevo como crioprotector externo puede variar dependiendo del tipo de crioprotector interno y del extensor con que se combine. Por ende, se recomienda evaluar la interacción entre la yema de huevo y el resto de los componentes del diluyente de crioconservación, que podrían estar inhibiendo su acción crioprotectora [39]. Asimismo, se recomienda evaluar otros parámetros de operación, cuya influencia sobre la calidad seminal ya ha sido comprobada en otras especies [21]. 
Figura 2. Evaluación de la fertilidad seminal post-congelación en Prochilodus mariae, empleando dos diluyentes de crioconservación ( $\mathrm{YH}=$ con yema de huevo; $\mathrm{SYH}=$ sin yema de huevo). . Control = semen fresco. Valores expresados como media $\pm \operatorname{SEM}(n=5)$. Entre barras, letras distintas indican diferencia estadística $(P<0,05)$.

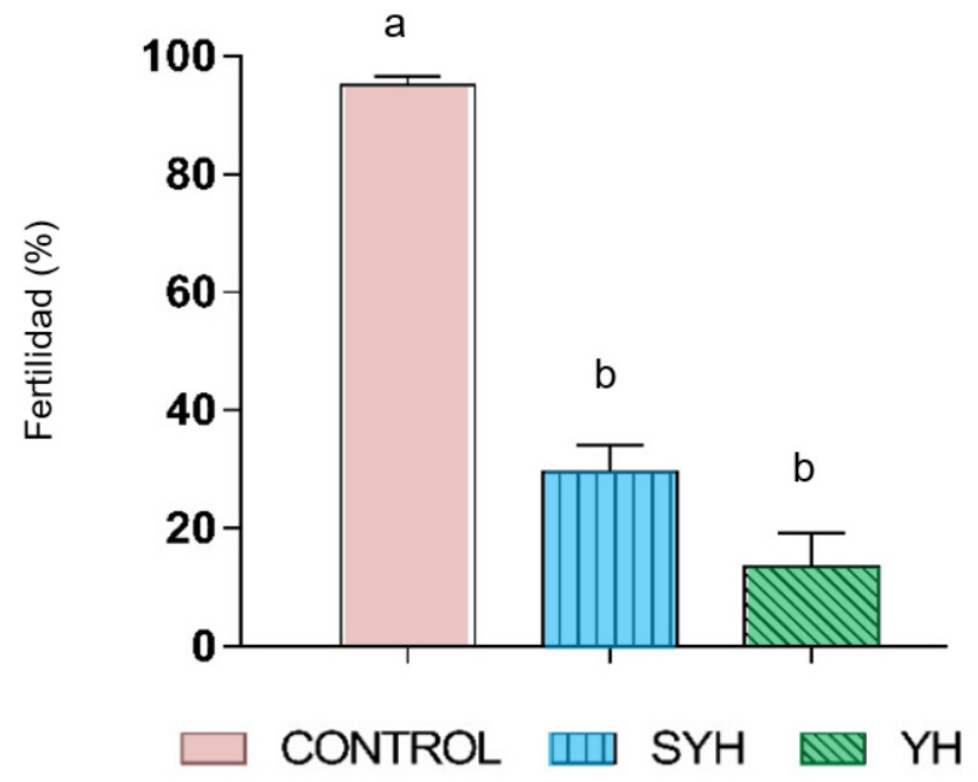

Se reportan investigaciones en las cuales la incorporación de la yema de huevo al diluyente de crioconservación, ha mejorado notoriamente la calidad seminal post-congelación en varias especies de peces. Algunas de las concentraciones de yema de huevo que permitieron el mejor rendimiento, son: $5 \%$ para salmón de río Brycon orbignyanus (Characiformes: Characidae) [39] y anguila europea Anguilla anguilla (Anguilliformes: Anguillidae) [40]; 7\% para trucha arcoíris Oncorhynchus mykiss (Salmoniformes: Salmonidae) [41, 42]; 10\% para bagre africano Clarias gariepinus (Siluriformes: Clariidae) [43], perca amarilla Perca flavescens (Perciformes: Percidae) y lucio del norte Esox lucius (Esociformes: Esocidae); 15\% para pez gato andador Clarias batrachus (Siluriformes: Clariidae) [44] y carpa común Cyprinus carpio (Cypriniformes: Cyprinidae) [45]. Sin embargo, aunque la incorporación de yema de huevo ha optimizado la calidad seminal post-congelación en estas especies, en otros peces como el salvelino Salvelinus alpinus (Salmoniformes: Salmonidae) y el aspio Aspius aspius (Cypriniformes: Cyprinidae), la calidad seminal post-congelación ha decrecido [44]. De igual modo, en cachama negra Colossoma macropomum (Characiformes: Serrasalmidae) se encontró que es mejor para la calidad seminal post-congelación omitir la yema de huevo al $12 \%$ que incorporarla en el diluyente de crioconservación [31].

Además, ya existen varios protocolos de crioconservación seminal de peces, en los cuales la yema de huevo está contenida dentro de la formulación del diluyente de crioconservación. En el protocolo para yamú Brycon amazonicus (Characiformes: Characidae) se establece una concentración de yema de huevo del 12\% v/v [23]; en el protocolo para salmón de río Brycon orbignyanus (Characiformes: Characidae) se establece una concentración del 5\% [46]; en el protocolo para Salmonidae se establece una concentración del 6 al 8\% p/v [47]; en el protocolo para cachama negra Colossoma macropomum (Characiformes: Serrasalmidae) se establece una concentración del 5\% [22]; en el protocolo para mero gigante Epinephelus lanceolatus (Perciformes: Serranidae) se establece una concentración del 5 al 10\% v/v [48]; en el protocolo para trucha arcoiris Salmo gairdneri (Salmoniformes: Salmonidae) se establece una concentración del 5 al 20\% v/v (preferiblemente al 10\%) [49]; en el protocolo para boga Leporinus obtusidens (Characiformes: Anostomidae) se establece una concentración del 10\% [50]1836. Asimismo, para el proceso de vitrificación de semen de esturión Acipenser spp. (Acipenseriformes: Acipenseri- 
dae) se establece una proporción de 8 a 12 partes de yema de huevo [51]. Con base en esto, se evidencia que en todos los protocolos e investigaciones citadas, la concentración de yema de huevo óptima varía con respecto a la especie con que se esté trabajando.

En cuanto a especies del género Prochilodus, Felizardo et al. [33] crioconservaron semen de sábalo jetón Prochilodus lineatus (Characiformes: Prochilodontidae) con un diluyente a base de yema de huevo al $5 \%$, metanol al $8 \%$ y BTS (Beltsville Thawing Solution $®$ ) al $5 \%$ y obtuvieron motilidades post-congelación superiores al $60 \%$ con una duración de más de 70 s y anormalidades post-congelación inferiores al 30\%. No obstante, cuando se modificó la composición del diluyente ( $8 \%$ yema de huevo, $10 \%$ metanol y $5 \%$ BTS) se evidenció un leve descenso de la motilidad al $50 \%$ y de la duración de la motilidad a 26 s [52]. Por su parte, Atencio et al. [53, 54] crioconservaron semen de bocachico Prochilodus magdalenae (Characiformes: Prochilodontidae) con un diluyente a base de yema de huevo al $12 \%$, glucosa al $5,5 \%$ y dimetil sulfóxido (DMSO) al 10\%, y obtuvieron valores promedio de motilidad post-congelación del $76 \%$ y una fertilidad del 70\%. Sin embargo, cuando sustituyeron el DMSO por dimetil acetamida (DMA) al $8 \%$, los valores de motilidad se redujeron al $31 \%$ y la fertilidad al $60 \%$. Con base en lo anterior, se evidencia que así se trabaje con la misma especie, el efecto de la yema de huevo sobre la calidad seminal post-congelación depende tanto del extensor y del crioprotector interno con que se combine.

En vista de que la concentración de yema de huevo establecida en cada protocolo de crioconservación varía conforme a la especie de pez con que se trabaje, y que el protocolo de crioconservación seminal para Prochilodus spp. todavía no ha sido estandarizado $[15,19,55]$ no es aconsejable crioconservar semen de P. mariae con diluyentes descritos en protocolos específicos para otras especies. Es necesario evaluar el efecto crioprotector de la yema de huevo a diferentes concentraciones, hasta encontrar el porcentaje óptimo para P. mariae. Las patentes europeas EP2043431B1 y ES2672630T3 establecen que la proporción de yema de huevo en el diluyente de crioconservación puede variar entre el 1 al 23\% de yema de huevo, independientemente de la especie [56, 57]; mientras que otros investigadores recomiendan un $20 \%$, en general $[58,59]$.

\section{CONCLUSIONES}

La yema de huevo no presentó el efecto crioprotector esperado, ya que los tratamientos con yema de huevo (YH) y sin yema de huevo (SYH) no exhibieron diferencias estadísticamente significativas entre sí en cuanto a su efecto sobre la integridad de membrana plasmática, la morfología y la fertilidad post-congelación en Prochilodus mariae. Por el contrario, al omitirse la yema de huevo se mejoró la motilidad y la duración de la motilidad post-congelación.

Se recomienda, evaluar diferentes proporciones de yema de huevo en el diluyente de crioconservación, antes de optar por retirarla de la formulación. Asimismo, es recomendable evaluar la interacción de la yema de huevo con otros componentes del diluyente de crioconservación, así como, la influencia de otros parámetros de operación.

\section{AGRADECIMIENTOS}

Los autores agradecen al Ministerio de Ciencia, Tecnología e Innovación de Colombia por la beca-pasantía del autor Astrid Stefanía Duarte-Trujillo. Asimismo, agradecen al fondo de Social de Educación Superior de la Gobernación del Meta (FSES) por la beca doctoral del autor Víctor Mauricio Medina-Robles. También agradecen al Instituto de Acuicultura - IALL, por el apoyo logístico y por prestar las instalaciones para el desarrollo de la investigación. 


\section{REFERENCIAS}

[1] SMITH, KEVIN G.; DARWALL, WILLIAM; BARRIOS, VIOLETA; NUMA, KATHERINE; ISFENDIYAROĞLU, SÜREYYA. Background to freshwater biodiversity in the eastern mediterranean. En: The status and distribution of freshwater biodiversity in the eastern Mediterranean. Cambridge (UK), Malaga (Spain) and Gland (Switzerland): International Union for Conservation of Nature (IUCN), 2014, p. 1-7.

[2] FREYHOF, JÖRG; BROOKS, EMMA. European Red List of Freshwater Fishes. Luxembourg (Luxemburgo): Office of the European Union, 2011, 70 p.

[3] VOLPEDO, ALEJANDRA; THOMPSON, GUSTAVO-ARIEL. Environmental changes on freshwater fish communities in South America in the last five decades: a case study in northeast Argentina. Sustainability, Agri, Food and Environmental Research, v. 4, n. 3, 2017, p. 47-61.

http://dx.doi.org/10.7770/safer-V4N3-art1047

[4] TEDESCO, PABLO A.; BEAUCHARD, OLIVIER; BIGORNE, RÉMY; BLANCHET, SIMÓN; BUISSON, LAËTITIA; CONTI, LORENZA; CORNU, JEAN-FRANÇOIS; DIAS, MURILO S.; GRENOUILLET, GAËL; HUGUENY, BERNARD; JÉZÉQUEL, CÉLINE; LEPRIEUR, FABIEN; BROSSE, SÉBASTIEN; OBERDORFF, THIERRY. Data Descriptor: A global database on freshwater fish species occurrence in drainage basins. Scientific Data, 4, 2017, p.1-6. https://doi.org/10.1038/sdata.2017.141

[5] REIS, R.E.; ALBERT, J.S.; DI DARIO, F.; MINCARONE, M.M.; PETRY, P.; ROCHA, L. A. Fish biodiversity and conservation in South America. Journal of Fish Biology, v. 89, n. 1, 2016, p. 12-47. https://doi.org/10.1111/jfb.13016

[6] MOJICA, J.I.E.; USMA-OVIEDO, J.U.E.; ALVAREZ-LEÓN, R.E.; LASSO, C.A. Libro rojo de peces dulceacuícolas de Colombia. Bogotá D.C. (Colombia): Instituto de Investigación de Recursos Biológicos Alexander von Humboldt, Instituto de Ciencias Naturales de la Universidad Nacional de Colombia, WWF Colombia y Universidad de Manizales, 2012, p. 154-159.

[7] ITALIA. ORGANIZACIÓN DE LAS NACIONES UNIDAS PARA LA ALIMENTACIÓN Y LA AGRICULTURA (FAO). Visión general del sector acuícola nacional - Colombia [online]. Disponible: http://www.fao.org/fishery/countrysector/naso_colombia/es\#tcN900B1 [citado 25 de enero de 2020].

[8] FRANCIA. ORGANIZACIÓN PARA LA COOPERACIÓN Y EL DESARROLLO ECONÓMICOS (OCDE). Pesca y acuicultura en Colombia. Bogotá D.C. (Colombia): 2016, 34 p.

[9] INSTITUTO COLOMBIANO AGROPECUARIO (ICA). Protección sanitaria de las especies acuícolas [online]. 2019. Disponible: https://www.ica.gov.co/getdoc/b082c759-18c7-47da-bed6-0ebe76b48fe0/acuicolas-(1).aspx [citado 25 de enero de 2020].

[10] RAMÍREZ-GIL, HERNANDO; AJIACO-MARTINEZ, ROSA; BARRETO-REYES, CARLOS. Tallas de captura de Prochilodus mariae en la parte alta del río Meta, Orinoquia colombiana. Orinoquia, v. 19, n. 2, 2015, p. 174-185. https://doi.org/10.22579/20112629.317.

[11] DO-NASCIMIENTO, CARLOS; HERRERA-COLLAZOS, EDGAR-ESTEBAN; HERRERA, GUIDO A.; ORTEGA-LARA, ARMANDO; VILLA-NAVARRO, FRANCISCO A.; USMA-OVIEDO, JOSÉ-SAULO; MALDONADO-OCAMPO, JAVIER A. Checklist of the freshwater fishes of Colombia: a Darwin Core alternative to the updating problem. ZooKeys, v. 708, 2017, p. 25-138.

10.3897 / zookeys.708.13897

[12] ORTEGA, JHOANA; YOSSA, MARTHA. Desempeño productivo del coporo con dietas isotópicas. Orinoquia (Suplemento), v. 18, n. 2, 2014, p. 278-285.

https://doi.org/10.22579/20112629.387

[13] SWITZERLAND. INTERNATIONAL UNION FOR CONSERVATION OF NATURE (IUCN). The IUCN red list of threatened species [online]. Disponible: https://www.iucnredlist.org/ [citado 10 de octubre de 2019].

[14] RAMIREZ-MERLANO, JUAN A.; MEDINA-ROBLES, VÍCTOR M.; CRUZ-CASALLAS, P.E. Crioconservación espermática en peces, un acercamiento en Siluriformes. Orinoquia, v. 14, n. 1, 2010, p. 59-71.

https://doi.org/10.22579/20112629.128 
[15] MARTÍNEZ-PÁRAMO, SONIA; ÁKOS, HORVÁTH; LABBÉ, CATHERINE; ZHANG, TIANTIAN; ROBLES, VANESA; HERRÁEZ, PAZ; SUQUET, MARC; ADAMS, SEREAN; VIVEIROS, ANA; TIERSCHK, TERRENCE; CABRITA, ELSA. Cryobanking of aquatic species. Aquaculture, v. 472, 2017, p. 156-177. https://doi.org/10.1016/j.aquaculture.2016.05.042

[16] CABRITA, E.; SARASQUETE, C.; MARTÍNEZ-PÁRAMO, S.; ROBLES, V.; BEIRÃO, J.; PÉREZ-CEREZALES ,V.; HERRÁEZ, M.P. Cryopreservation of fish sperm: Applications and perspectives. Journal of Applied Ichthyology, v. 26, n. 5, 2010, p. 623-635. https://doi.org/10.1111/j.1439-0426.2010.01556.x

[17] MARTÍNEZ, JOSÉ-GREGORIO; ATENCIO-GARCIA, VÍCTOR; PARDO-CARRASCO, SANDRA. DNA fragmentation and membrane damage of bocachico Prochilodus magdalenae (Ostariophysi: Prochilodontidae) sperm following cryopreservation with dimethylsulfoxide and glucose. Neotropical Ichthyology, v. 10, n. 3, 2012, p. 577-586. https://doi.org/10.1590/S1679-62252012005000018

[18] CABRITA, E.; MARTÍNEZ-PARAMO, S.; GAVAI, P.J.; RIESCO, M.F.; VALCARCE, D.G; SARASQUETE, C.; HERRÁEZ, M.P.; ROBLES, V. Factors enhancing fish sperm quality and emerging tools for sperm analysis. Aquaculture, v. 432, 2014, p. 389-401. https://doi.org/10.1016/j.aquaculture.2014.04.034

[19] MARTÍNEZ, J.G.; TARAZONA-MORALES, A.M.; PARDO-CARRASCO, S.C. Sperm cryopreservation of freshwater fish bocachico (Prochilodus magdalenae) in DMSO and glucose and its effects on fertilization and hatching efficiency. Animal Reproduction, v. 9, n. 1, 2012, p. 19-26.

[20] BENSON, JAMES D. Modeling and Optimization of Cryopreservation. En: Cryopreservation and Freeze-Drying Protocols. Hertfordshire (UK): Humana Press, 2015, p. 83-120. https://doi.org/10.1007/978-1-4939-2193-5_3

[21] VIVEIROS, A.T.M.; GODINHO, H.P. Sperm quality and cryopreservation of Brazilian freshwater fish species: A review. Fish Physiology and Biochemistry, v. 35, n. 1, 2009, p. 137-150. https://doi.org/10.1007/s10695-008-9240-3

[22] CARNEIRO, PAULO; AZEVEDO, HYMERSON C.; SANTOS, JADSON P.; ALEXANDRE, MARIA. Cryopreservation of tambaqui (Colossoma macropomum) semen: Extenders, cryoprotectants, dilution ratios and freezing methods. CryoLetters, v. 33, n. 5, 2012, p. 385-393.

[23] CRUZ-CASALLAS, PABLO; MEDINA-ROBLES, VÍTOR; VELASCO-SANTAMARÍA, YOHANA. Protocolo para la crioconservación de semen de yamú (Brycon amazonicus Spix \& Agassiz 1829). Revista Colombiana de Ciencias Pecuarias, v. 19, n. 2, 2006, p. 146-151.

[24] PARDO-CARRASCO, SANDRA; ARIAS-CASTELLANOS, JOSÉ; SUÁREZ-MAHECHA, HÉCTOR; CRUZ-CASALLAS, PABLO; VÁSQUEZ-TORRES, WALTER; ATENCIO-GARCÍA, VÍCTOR; ZANIBONI-FILHO, EVOY. Inducción a la maduración final y ovulación del yamú Brycon amazonicus con EPC y mGnRH-a. Revista Colombiana de Ciencias Pecuarias, v. 19, n. 2, 2006, p. 160-166.

[25] AYA, ELIZABETH; ARIAS, JOSÉ. Reproducción inducida de Pimelodus pictus con extracto de hipófisis de carpa (EHC) y Ovaprim ${ }^{\circledR}$ Induced spawning of Pimelodus pictus with carp hypophysis. Revista MVZ Córdoba, v. 6, n. 1, 2011, p. 2317-2323.

[26] CRUZ-CASALLAS, PABLO; LOMBO-RODRÍGUEZ, DORA; VELASCO-SANTAMARÍA, YOHANA-MARÍA. Milt quality and spermatozoa morphology of captive Brycon siebenthalae (Eigenmann) broodstock. Aquaculture Research, v. 36, n. 7, 2005, p. 682-686. https://doi.org/10.1111/j.1365-2109.2005.01273.x

[27] RAMIREZ-MERLANO, JUAN-ANTONIO; VELASCO-SANTAMARÍA, YOHANA-MARÍA; MEDINA-ROBLES, VÍCTOR-MAURICIO; CRUZ-CASALLAS, PABLO-EMILIO. Cryopreservation effects on the sperm quality of cachama blanca Piaractus brachypomus (Cuvier 1818). Aquaculture Research, v. 42, n. 6, 2011, p. 738-745. https://doi.org/10.1111/j.1365-2109.2011.02835.x

[28] CRUZ-CASALLAS, PABLO-EMILIO; MEDINA-ROBLES, VÍCTOR; VELASCO-SANTAMARÍA, YOHANA-MARÍA. Evaluación de diferentes crioprotectores para la crioconservación de espermatozoides de yamú (Brycon amazonicus). Revista Colombiana de Ciencias Pecuarias, v. 19, n. 2, 2006, p. 152-159. 
[29] MEDINA-ROBLES, VÍCTOR-MAURICIO; CRUZ-CASALLAS, PABLO-EMILIO. Efecto del volumen de empaque sobre la tasa de congelación- descongelación y la fertilidad de semen crioconservado de yamú (Brycon amazonicus). Archivos de medicina veterinaria, v. 39,n. 3, 2007, p. 229-237. http://dx.doi.org/10.4067/S0301-732X2007000300006

[30] VELASCO-SANTAMARÍA, YOHANA-MARÍA; MEDINA-ROBLES, VÍCTOR-MAURICIO; CRUZ-CASALLAS, PABLO-EMILIO. Cryopreservation of yamú (Brycon amazonicus) sperm for large scale fertilization. Aquaculture, v. 256, n. (1-4), 2006, p. 264-271. https://doi.org/10.1016/j.aquaculture.2006.02.039

[31] MEDINA-ROBLES, VÍCTOR-MAURICIO; GUAJE-RAMÍREZ, DIANA; MARIN-COSSIO, LAURA; SANDOVAL-VARGAS, LEYDY; CRUZ-CASALLAS, PABLO-EMILIO.Crioconservación seminal de Colossoma macropomum como estrategia de producción y conservación en la Orinoquia Colombiana. Orinoquia, v. 23, n. 1, 2019, p. 15-24. http://dx.doi.org/10.22579/20112629.537

[32] VIVEIROS, A.T.M.; MARIA, A.N. Semen cryopreservation of piracanjuba (Brycon orbignyanus), an endangered Brazilian species. En: Methods in Reproductive Aquaculture: Marine and Freshwater Species. Boca Ratón (USA): CRC Press, 2008, p. 361-365. https://doi.org/10.1201/9780849380549-27

[33] FELIZARDO, V.D.O.; MELLO, R.A.; MURGAS, L.D.S.; ANDRADE, E.S.; DRUMOND, M.M.; ROSA, P.V. Effect of cryopreservant combinations on the motility and morphology of curimba (Prochilodus lineatus) sperm. Animal reproduction science, v. 122, n. (3-4), 2010, p. 259-263. https://doi.org/10.1016/j.anireprosci.2010.08.020

[34] MILIORINI, ALÉSSIO-BATISTA; SOLIS-MURGAS, LUIS-DAVID; VIEIRA-ROSA, PRISCILA; OBERLENDER, GUILHERME; MACHADO-PEREIRA, GILMARA-JUNQUEIRA; VICENTE-DA COSTA, DIEGO. A morphological classification proposal for curimba (Prochilodus lineatus) sperm damages after cryopreservation. Aquacuaculture Research, v. 42, n. 2, 2011, p. 177-187. https://doi.org/10.1111/j.1365-2109.2010.02575.x

[35] PÉREZ-CEREZALES, S.; MARTÍNEZ-PÁRAMO,S.; BEIRÃO, J.; PAEZ-HERRÁEZ, MARÍA. Evaluation of DNA damage as a quality marker for rainbow trout sperm cryopreservation and use of LDL as cryoprotectant. Theriogenology, v. 74, n. 2, 2010, p. 282-289. https://doi.org/10.1016/j.theriogenology.2010.02.012

[36] DONG, XIANG-DONG; RODENBURG, SARAH; HILL, DANA; VANDERVOORT, CATHERINE. The role of low-density lipoprotein (LDL) and high-density lipoprotein (HDL) in comparison with whole egg yolk for sperm cryopreservation in rhesus monkeys. Asian journal of andrology, v. 13, n. 3, 2011, p. 459-464. https://doi.org/10.1038/aja.2010.145

[37] FARLEY, JANE; OSTERMEIER, CHARLES; TAFT, ROBERT; WILES, MICHAEL V. Sperm cryoprotective media. AU 2012203918 A1. Melbourne (Australia): 2012.

[38] DIWAN, A.D.; AYYAPPAN, S.; LAL, K.K.; LAKRA, W.S. Cryopreservation of fish gametes and embryos. The Indian Journal of Animal Sciences, v. 80, n. 4, 2010, p.109-124.

[39] ALEXANDRE-NÍZIO, MARÍA; VIVEIROS, ANNA; FONSECA-DE FREITAS, RILKE-TADEU; OLIVEIRA, ALEXMILIANO V. Extenders and cryoprotectants for cooling and freezing of piracanjuba (Brycon orbignyanus) semen, an endangered Brazilian teleost fish. Aquaculture, v. 260, 2006, p. 298-306. https://doi.org/10.1016/j.aquaculture.2006.06.011

[40] HERRANZ-JUSDADO, JUAN-GERMAN; GALLEGO, VÍCTOR; ROZENFELD, CHRISTOFFER; MORINI, MARÍA; PÉREZ, LUZ; ASTURIANO, JUAN. European eel sperm storage: optimization of short-term protocols and cryopreservation of large volumes. Aquaculture, v. 506, 2019, p. 42-50. https://doi.org/10.1016/j.aquaculture.2019.03.019

[41] LAHNSTEINERL, F.; BERGER,B.; WEISMANN,T.; PATZNER, R.The influence of various cryoprotectants on semen quality of the rainbow trout. Journal of Applied Ichthyology, v. 12, 1996, p. 99-106.

https://doi.org/10.1111/j.1439-0426.1996.tb00070.x 
[42] LAHNSTEINER, FRANZ. Semen cryopreservation in the Salmonidae and in the Northern pike. Aquacuaculture Research, v. 31, n. 3, 2000, p. 245-258. https://doi.org/10.1046/j.1365-2109.2000.00452.x

[43] RURANGWA, EUGÉNE; VOLCKAERT, FILIP; HUYSKENS, GEERT; KIME, DAVID E.; FRANS, OLLIVIER. Quality control of refrigerated and cryopreserved semen using computer-assisted sperm analysis (CASA), viable staining and standardized fertilization in African catfish (Clarias gariepinus). Theriogenology, v. 55, n. 3, 2001, p. 751-769. https://doi.org/10.1016/S0093-691X(01)00441-1

[44] MUCHLISIN, Z.A. Current Status of extenders and cryoprotectants on fish spermatozoa cryopreservation. Biodiversitas, v. 6, n. 1, 2005, p. 66-69. https://doi.org/10.13057/biodiv/d060114

[45] BOZKURT, YUSUFUT; YAVAS,ILKER; CENGIZ, YILDIZ. Effect of different avian egg yolk types on fertilization ability of cryopreserved common carp (Cyprinus carpio) spermatozoa. Aquaculture international, v. 22, 2014, p. 131-139. https://doi.org/10.1007/s10499-013-9728-4

[46] VIVIANE-DE OLIVEIRA, FELIZARDO; VIEIRA-MELO, CARLOS-CICINATO; SOLIS-MURGAS, LUIS-DAVID; DE SOUZA-ANDRADE, ESTEFÂNIA; NAVARRO, RODRIGO-DIANA; FONSECA-DE FREITAS, RILKE-TADEU. Optimization of artificial propagation in piracanjuba fish Brycon orbignyanus using cryopreserved semen. CryoLetters, v. 37, n. 5, 2016, p. 330-334.

[47] ALEANDRI, RICARDO; GALLI, ANDREA. Method for cryopreservation of teleostei semen. CA 2.388.741 A1, Classification A01N1/02. Gatineau (Canadá): 2001.

[48] LIU, Q. et al. A kind of sub- Large Copacity of epinephelus lanceolatus milt, the method for high-efficiency ultralow temperature freezen protective. CN 105.532.639 B, Classification A01N1/0221. Qingdao (China): 2018.

[49] BAYNES, STHEPEN M.; SCOTT, ALEXANDER. Cryopreservation of rainbow trout spermatozoa: The influence of sperm quality, egg quality and extender composition on post-thaw fertility. Aquaculture, v. 66, n. 1, 1987, p. 53-67. https://doi.org/10.1016/0044-8486(87)90283-3

[50] TAITSON, PAULO-FRANCO; CHAMI, E.; PEREIRA-GODHINO, HELOISA.Gene banking of the neotropical fish Leporinus obtusidens (Valenciennes, 1836): A protocol to freeze its sperm in the field. Animal reproduction science, v. 105, n. (3-4), 2008, p. 283-291. https://doi.org/10.1016/j.anireprosci.2007.03.009

[51] ZHAO, Z.; ZHAO, W. Vitrification freezing method for sturgeon sperms. CN 103.931.607 A, Classification A01N1/02. Hengyang (China): 2014.

[52] VASCONCELOS-NOGUEIRA, ANA-CARINA; SOUZA-DE CARVALHO, ALINE-FERREIRA; FREITAS-GARCIA, RAYCON-ROBERTO; ELIZIANNE-RAMOS, SHAYENNE; SOLIS-MURGAS, LUIS-DAVID. Cryopreservation of prochilodus lineatus semen: Effect of cryoprotectants combination. Boletim do Instituto de Pesca, v. 41, 2015, p. 817-824. https://doi.org/10.20950/1678-2305.2015v41nep817

[53] ATENCIO-GARCÍA, VÍCTOR; SPINOSA, JOSÉ; MARTÍNEZ, JOSÉ; PARDO-CARRASCO, SANDRA. Insemination of bocachico fish (Prochilodus magdalenae) with fresh or cryopreserved semen: effect of spermatozoa/oocyte ratio. Revista Colombiana de Ciencias Pecuarias, v. 28, 2015, p. 347-355. http://dx.doi.org/10.17533/udea.rccp.v28n4a07

[54] ATENCIO-GARCÍA, VÍCTOR; MARTÍNEZ, JOSÉ; PARDO-CARRASCO, SANDRA. Evaluación de dimetilacetamida como crioprotector para la crioconservación de semen de bocachico Prochilodus magdalenae. Archivos de medicina veterinaria, v. 45, 2013, p. 151-158. http://dx.doi.org/10.4067/S0301-732X2013000200006

[55] ASTURIANO-NEMESIO, JUAN-FRANCISCO; CABRITA, ELSA; HORVÁTH, ÁKOS. Progress, challenges and perspectives on fish gamete cryopreservation: A mini-review. General and comparative endocrinology, v. 245, 2016, p. 69-76.

https://doi.org/10.1016/j.ygcen.2016.06.019 
[56] OSTERMEIER, G.C. et al. Sperm cryoprotective media. EP 2.043.431 B1, Classification A01N1/0221. Múnich (Alemania): 2018.

[57] OSTERMEIER, G.C. et al. Sperm cryoprotectants. ES 2.672.630 T3, Classification A01N1/0221. Madrid (España): 2018.

[58] HERICKHOFF, L.A. et al. System and substances for cryopreservation of viable cells. US 20.150.037.783 A1, Classification C12N5/0693. Fort Collins (USA): 2015.

[59] HERICKHOFF, L.A. et al. Methods and systems for synergistic continuity approaches to treatment and preservation of biological cells. WO 2.019.104.184 A1, Classification A01N1/0226. Fort Collins (USA): 2019. 